\title{
Atitudes e conhecimentos de técnicos de enfermagem sobre cuidados a pacientes
}

\section{com transtornos mentais}

Zilda Maria de Melo ${ }^{1}$, Natália Priolli Jora Pegoraro ${ }^{2}$, Manoel Antônio dos Santos ${ }^{3}$, Sandra Cristina Pillon ${ }^{4}$

\author{
${ }^{1}$ Enfermeira, Mestre em Tecnologia e \\ Inovação em Enfermagem. Universidade \\ de Franca. Franca, SP, Brasil. E-mail: \\ melozilda@usp.br. \\ ${ }^{2}$ Enfermeira, Doutora em Enfermagem \\ Psiquiátrica. Enfermeira Coordenadora do \\ Centro de Atenção Psicossocial de de \\ Ribeirão Preto. Ribeirão Preto, SP, Brasil. E- \\ mail: natalia.jora@usp.br. \\ ${ }^{3}$ Psicólogo, Doutor em Psicologia Clínica. \\ Professor Associado da Faculdade de \\ Filosofia Ciências e Letras de Ribeirão Preto \\ da Universidade de São Paulo (USP). \\ Ribeirão Preto, SP, Brasil. E-mail: \\ masantos@ffclrp.usp.br. \\ ${ }^{4}$ Enfermeira, Doutora em Psiquiatria e \\ Psicologia Médica. Professor Associado da \\ Escola de Enfermagem de Ribeirão \\ Preto/USP. Ribeirão Preto, SP, Brasil. E- \\ mail: pillon@eerp.usp.br.
}

Recebido: 24/09/2015.

Aceito: 30/11/2015.

Publicado: 31/03/2016.

Como citar esse artigo:

Melo ZM, Pegoraro NPJ, Santos MA, Pillon SC. Atitudes e conhecimentos de técnicos de enfermagem sobre cuidados a pacientes com transtornos mentais. Rev. Eletr. Enf. [Internet]. 2016 [acesso em:

____;18:e1141. Disponível em: http://dx.doi.org/10.5216/ree.v18.37746.

\section{RESUMO}

O estudo teve por objetivo avaliar atitudes e conhecimentos teóricopráticos de técnicos de enfermagem sobre cuidados a pacientes com transtornos mentais. Estudo descritivo realizado em um pronto socorro municipal do interior paulista. Informações sociodemográficas e formação profissional, atitudes e conhecimentos sobre identificação de sinais e sintomas, sentimentos e assistência de enfermagem a pessoas com transtornos mentais foram avaliadas em 69 técnicos de enfermagem, caracterizados por serem do sexo feminino, adultos, com ensino médio e nível superior. A maioria recebeu conteúdo teórico sobre como identificar sinais e sintomas, porém menos da metade recebeu informações sobre a sistematização da assistência de enfermagem. Apesar de sentirem muito interesse na área de psiquiatria, os profissionais percebiam esses pacientes como indivíduos imprevisíveis, necessitado de cuidados constantes. Constatou-se a necessidade de melhores investimentos em temas relacionados aos cuidados de enfermagem para que os profissionais possam oferecer uma assistência de melhor qualidade as pessoas com transtornos mentais.

Descritores: Transtornos Mentais; Cuidados de Enfermagem; Conhecimentos, Atitudes e Prática em Saúde.

\section{INTRODUÇÃO}

Uma das maiores barreiras para o desenvolvimento de propostas terapêuticas mais eficazes em saúde mental nos serviços de saúde nos países em desenvolvimento tem sido o número insuficiente de profissionais 
devidamente capacitados e supervisionados para oferecerem cuidados de saúde mental ${ }^{(1)}$.

A qualificação em saúde mental muitas vezes têm sido negligenciada por parte do poder público e também pelos profissionais de enfermagem, o que contribui para a assistência pouco especializada, inadequada e de baixa qualidade nos serviços de atenção primária à saúde ${ }^{(1)}$. Essa deficiência de incorporação de conhecimentos qualificados por parte dos profissionais pode estar associada à atitudes desfavoráveis a uma assistência de qualidade. A atitude uma vez que é definida como uma propensão à ação, exprime o que o profissional pensa, sente e age em relação a uma determinada situação ou objeto ${ }^{(2)}$. Além disso, temas relacionados à saúde mental tendem a mobilizar os profissionais em termos afetivos, suscitando uma série de dificuldades e precariedades na assistência junto aos usuários dos serviços de saúde ${ }^{(1-3)}$.

Estudo de revisão sistemática acerca das atitudes de estudantes de enfermagem em relação à saúde mental evidenciou que essa é uma área de baixa preferência pelos estudantes para seguir e ter uma carreira bem-sucedida. O estudo também destacou a influência do ensino nas atitudes desenvolvidas no decorrer da formação, sugerindo que essas eram mais favoráveis quando os estudantes haviam recebido mais horas de preparação teórica e prática ${ }^{(4)}$.

Com o foco na formação de profissionais de enfermagem, a Organização Mundial de Saúde (OMS) em 2008 disponibilizou um relatório destacando a importância da integração da saúde mental na atenção primária e delineando as competências necessárias, os conhecimentos específicos e a prática de habilidades profissionais em diversos países ${ }^{(5)}$. Esse relatório teve por objetivo facilitar e ampliar a percepção da necessidade dessa integração na assistência em serviços de saúde. Investimentos neste nível são importantes, pois o estilo clínico adquirido na etapa da formação profissional e técnica tende a se manter relativamente fixo no decorrer da atuação profissional ${ }^{(2)}$.

Investimentos na formação do profissional em cursos técnicos e de graduação podem favorecer atitudes positivas em relação à assistência em saúde mental. Entende-se que o processo de aprendizagem promove e valoriza as atitudes positivas, pois refletem o estado interno do indivíduo e afetam suas escolhas, ações ou comportamentos frente a um determinado objeto ${ }^{(6-7)}$.

O conhecimento sobre as atitudes de prestadores de cuidados vem sendo valorizado como uma variável importante do tratamento e é um potencial determinante da qualidade dos cuidados ${ }^{(2,7)}$. Atitudes positivas facilitam o desenvolvimento da relação terapêutica, sendo um componente necessário de sucesso, como mostram os estudos que têm focalizado a relação terapêutica entre profissional e cliente. As atitudes de profissionais de enfermagem frente aos problemas de saúde mental se correlacionam com a quantidade de conhecimento incorporado sobre o tema ${ }^{(2,4,7)}$. Assim, o profissional desenvolve a assistência em enfermagem baseado em seus conhecimentos relacionados à saúde mental, que por sua vez orientam o desenvolvimento de suas crenças e atitudes, que irão impactar na qualidade dos cuidados ${ }^{(2)}$.

Evidência mostra que profissionais de enfermagem de unidades psiquiátricas hospitalares, tendem a concentrar sua assistência mais focadas nas necessidades físicas. No entanto, a atenção aos aspectos psíquicos e emocionais dos pacientes tem ficado aquém do desejável em suas avaliações ${ }^{(8)}$. Estudos reforçam 
que os profissionais da equipe de enfermagem, de um modo geral, não se sentem à vontade em cuidar de pessoas com TM, seja pela falta de conhecimentos específicos sobre o assunto, seja pelo fato de terem recebido uma formação focada no modelo $\operatorname{asilar}^{(9-10)}$.

Todavia, existe atualmente uma intensa preocupação com a formação e atuação do técnico de enfermagem que atua em serviços de saúde, principalmente nos serviços de urgência e emergência psiquiátrica. Diversos questionamentos têm sido levantados em relação aos motivos que levaram esses profissionais a atuarem na área, as dificuldades encontradas na sua atuação, a educação continuada recebida em serviço diante da necessidade de integrar novos conhecimentos, assim como dificuldades relacionadas à atuação profissional e ao planejamento para o treinamento na assistência de enfermagem ${ }^{(11)}$. Mediante esses questionamentos, o presente estudo teve por objetivo avaliar atitudes, conhecimentos, percepções e sentimentos de técnicos de enfermagem que atuam em uma unidade de urgência e emergência sobre os cuidados às pessoas com Transtornos Mentais.

\section{MATERIAIS E MÉTODOS}

Estudo descritivo de abordagem quantitativa, realizado em um Pronto Socorro Municipal (PSM) de um município de médio porte localizado no interior do Estado de São Paulo, Brasil, que atende todas as demandas de urgência e emergência relacionadas às doenças de um modo geral, incluindo os transtornos psiquiátricos. O estudo foi realizado de fevereiro de 2013 a julho de 2015.

No momento da coleta de dados, a equipe do referido serviço estava composta por 89 técnicos de enfermagem que trabalhavam nos três períodos no Pronto Socorro Municipal (PSM). Todos foram convidados a participar do estudo, sendo que, 69 (77,5\%) técnicos de enfermagem aceitaram colaborar, compondo a amostra. Os critérios de elegibilidade foram: ser funcionário vinculado a instituição, de ambos os sexos, com idade igual ou superior a 18 anos. Os critérios de exclusão foram: estar em afastamento do serviço por quaisquer motivos. Os participantes foram recrutados durante as atividades desenvolvidas no local de trabalho, respeitando-se a disponibilidade de cada profissional.

Para a coleta de dados foi utilizado um questionário composto por 1 - Informações sociodemográficas: idade, sexo, escolaridade, estado civil, dados relacionados à formação acadêmica e ao trabalho na instituição, tempo de atuação na Enfermagem, no PSM e na mesma equipe. 2 - Sentimentos dos profissionais frente à assistência aos pacientes com TM: um check-list com 27 itens ${ }^{(12)} .3$ - Escala de atitudes, conhecimentos e práticas $^{(2)}$ em relação à pessoa com TM e ao trabalho em saúde mental ${ }^{(13)}$ : com 25 itens, que avaliam conhecimentos, atitudes e práticas dos profissionais no desempenho de suas atividades assistenciais junto a pacientes com $\mathrm{TM}^{(2)}$.

O projeto foi aprovado pelo Comitê de Ética em Pesquisa da Escola de Enfermagem de Ribeirão Preto da Universidade de São Paulo, Processo $\mathrm{n}^{\circ}$ 704.097, de acordo com o preconizado pelas normas brasileiras para pesquisas com seres humanos.

Para análise dos dados foi elaborado um banco de dados no Statistical Program of Social Science (SPSS) 
versão 19, for Windows. A análise descritiva foi realizada por meio cálculo de médias, frequências e porcentagens das variáveis, visando à elucidação das características da amostra.

\section{RESULTADOS}

A amostra foi composta por 69 profissionais de enfermagem, caracterizados por serem predominantemente do sexo feminino $(75,4 \%)$, adultos $(36,2 \%)$, casados $(46,4 \%)$, com Ensino Médio completo (47,8\%), que não possuíam outro emprego $(60,9 \%)$ (Tabela 1$)$.

Tabela 1: Informações sociodemográficas de técnicos de enfermagem que atuam em um PSM ( $n=69$ ). Franca, SP, Brasil, 2015.

\begin{tabular}{|c|c|c|c|}
\hline & & $\mathbf{n}$ & $\%$ \\
\hline \multirow{3}{*}{ Sexo } & Masculino & 17 & 24,6 \\
\hline & Feminino & 52 & 75,4 \\
\hline & Casado & 32 & 46,4 \\
\hline \multirow[t]{3}{*}{ Estado civil } & Solteiro & 27 & 39,1 \\
\hline & Separado/Divorciado & 10 & 14,5 \\
\hline & $22-29$ anos & 20 & 29,0 \\
\hline \multirow[t]{3}{*}{ Faixa etária } & $30-39$ anos & 24 & 34,8 \\
\hline & $40-59$ anos & 25 & 36,2 \\
\hline & Ensino Fundamental & 2 & 2,9 \\
\hline \multirow[t]{2}{*}{ Escolaridade } & Ensino Médio & 33 & 47,8 \\
\hline & Ensino Superior (Enfermagem) & 32 & 46,4 \\
\hline \multirow{3}{*}{ Possui outro emprego } & Sim & 26 & 37,7 \\
\hline & Não & 42 & 60,9 \\
\hline & $\leq 1$ ano & 15 & 21,7 \\
\hline \multirow{4}{*}{ Tempo de trabalho na Enfermagem (em anos) } & $2-5$ anos & 31 & 44,9 \\
\hline & $6-11$ anos & 10 & 14,5 \\
\hline & $12-21$ anos & 12 & 17,4 \\
\hline & $\leq 1$ ano & 18 & 26,1 \\
\hline \multirow[t]{3}{*}{ Tempo de trabalho no PSM (em anos) } & $2-5$ anos & 27 & 39,1 \\
\hline & $\geq 6$ anos & 12 & 17,4 \\
\hline & $\leq 1$ ano & 22 & 31,9 \\
\hline \multirow[t]{2}{*}{ Tempo de atuação na mesma equipe (em anos) } & $2-5$ anos & 30 & 43,5 \\
\hline & $\geq 6$ anos & 13 & 18,8 \\
\hline
\end{tabular}

Em relação à educação formal recebida, os profissionais consideraram que têm grande interesse em participar do processo de educação (dados não apresentados em tabela).

A maioria relatou que recebeu informações sobre os problemas físicos e TM $(88,4 \%)$, tratamentos dos problemas psiquiátricos (82\%), problemas familiares nos TM $(81,2 \%)$ e identificação de sinais e sintomas dos TM (69,6\%). Apenas 49,3\% respondeu que tinha recebido informações sobre o desenvolvimento da Sistematização da Assistência de Enfermagem durante a formação (Tabela 2). 
Tabela 2: Informações recebidas pelos profissionais de enfermagem sobre saúde mental durante a formação profissional $(n=69)$. Franca, SP, Brasil, 2015.

\begin{tabular}{|c|c|c|}
\hline \multirow{2}{*}{ Informações recebidas } & \multicolumn{2}{|c|}{ Sim } \\
\hline & $\mathbf{n}$ & $\%$ \\
\hline Problemas físicos e TM & 61 & 88,4 \\
\hline Tratamento dos problemas psiquiátricos & 57 & 82,6 \\
\hline Problemas familiares e TM & 56 & 81,2 \\
\hline TM em adolescentes ou pessoas idosas & 49 & 71,0 \\
\hline TM em pessoas idosas & 45 & 70,3 \\
\hline Identificação de sinais e sintomas dos TM & 48 & 69,6 \\
\hline Acidentes (ocupacionais, trânsito) e TM & 42 & 60,9 \\
\hline Obtenção detalhada da história do paciente com TM & 38 & 55,1 \\
\hline TM em gestantes & 35 & 50,7 \\
\hline Desenvolvimento da Sistematização da Assistência de Enfermagem (SAE) & 34 & 49,3 \\
\hline TM como causadores da diminuição da produtividade no local de trabalho & 33 & 47,8 \\
\hline Avaliação das opções de tratamento & 32 & 46,4 \\
\hline Barreiras no diagnóstico e tratamento (meios que impedem o médico, paciente e a família de intervir) & 21 & 30,4 \\
\hline
\end{tabular}

Em relação aos cuidados e tratamento às pessoas com TM, quase a totalidade concorda que os cuidados de enfermagem em saúde mental deveriam estar integrados nos cuidados de saúde geral (96,9\%) e que os profissionais deveriam receber treinamento para identificar sinais e sintomas de TM (96,9\%). Sobre a percepção em relação às pessoas com TM, a maioria concordou que são normalmente imprevisíveis $(95,3 \%)$, necessitam de cuidados constantes $(90,6 \%)$, mas podem levar uma vida normal $(78,1 \%)$. O que chama a atenção é o fato de 59,4\% concordarem que pessoas com TM são normalmente perigosos. Quanto à percepção acerca do trabalho em saúde mental, apenas metade dos profissionais de enfermagem se sente confortável em atender pessoas com TM $(50,1 \%)$ (Tabela 3$)$.

Tabela 3: Percepções em relação a cuidados e tratamento, pessoa com TM e trabalho em saúde mental, segundo os técnicos de enfermagem que atuam em um PSM ( $n=69)$. Franca, SP, Brasil, 2015.

\begin{tabular}{|c|c|c|c|c|c|c|c|}
\hline & \multirow{2}{*}{ Percepções } & \multicolumn{2}{|c|}{ Discordo } & \multicolumn{2}{|c|}{ Indiferente } & \multicolumn{2}{|c|}{ Concordo } \\
\hline & & $\mathbf{n}$ & $\%$ & $\mathbf{N}$ & $\%$ & $\mathbf{N}$ & $\%$ \\
\hline \multirow[b]{2}{*}{$\begin{array}{l}\text { Cuidados e } \\
\text { Tratamento }\end{array}$} & $\begin{array}{l}\text { Cuidados de Enfermagem em saúde mental deveriam estar } \\
\text { integrados nos cuidados de saúde geral }\end{array}$ & 2 & 3,1 & - & - & 62 & 96,9 \\
\hline & $\begin{array}{l}\text { Profissionais de unidades de emergência deveriam ser treinados } \\
\text { para identificar sintomas e realizar cuidados de enfermagem a } \\
\text { paciente com TM }\end{array}$ & 1 & 1,6 & - & - & 62 & 96,9 \\
\hline \multirow{7}{*}{$\begin{array}{l}\text { Pessoas com } \\
\text { TM }\end{array}$} & Medicações são efetivas no tratamento de doenças mentais & 3 & 4,7 & 2 & 3,1 & 56 & 87,5 \\
\hline & São normalmente imprevisíveis & - & - & 2 & 3,1 & 61 & 95,3 \\
\hline & Necessitam de cuidados constantemente & 3 & 4,7 & - & - & 58 & 90,6 \\
\hline & Podem levar uma vida normal & 8 & 12,5 & 4 & 6,3 & 50 & 78,1 \\
\hline & São normalmente perigosas & 19 & 29,7 & 6 & 9,4 & 38 & 59,4 \\
\hline & São normalmente violentas & 26 & 40,7 & 13 & 20,3 & 24 & 37,5 \\
\hline & $\begin{array}{l}\text { Deveriam ser capazes de receber tratamento no mesmo centro } \\
\text { de saúde das pessoas com doença física }\end{array}$ & 42 & 65,6 & 4 & 6,3 & 16 & 25,0 \\
\hline \multirow{4}{*}{$\begin{array}{l}\text { Trabalho em } \\
\text { Saúde } \\
\text { Mental }\end{array}$} & $\begin{array}{l}\text { Deveriam ser confinadas em locais ou hospitais para o resto de } \\
\text { suas vidas }\end{array}$ & 55 & 86,0 & 4 & 6,3 & 3 & 4,7 \\
\hline & Eu me sinto confortável em atender pessoas com doença mental & 21 & 32,8 & 9 & 14,1 & 32 & 50,1 \\
\hline & $\begin{array}{l}\text { O hospital é o local mais apropriado da região para as pessoas } \\
\text { com doença mental morar }\end{array}$ & 25 & 39,0 & 11 & 17,2 & 26 & 40,6 \\
\hline & É difícil conversar com alguém com problemas mentais & 32 & 50,0 & 8 & 12,5 & 22 & 34,4 \\
\hline
\end{tabular}


Os profissionais de enfermagem apresentaram tanto sentimentos positivos $(52,2 \%$ compaixão e $26,1 \%$ aceitação), como negativos ( $42 \%$ insegurança e $40,6 \%$ tristeza) em relação aos pacientes com TM. Um dado digno de atenção é que a quantidade de sentimentos negativos evocados prevaleceu em comparação aos positivos (Tabela 4).

Tabela 4: Sentimentos expressados em relação aos pacientes com TM, segundo os técnicos de enfermagem que atuam em um PSM ( $\mathrm{n}=69)$. Franca, SP, Brasil, 2015.

\begin{tabular}{|c|c|c|c|}
\hline & & \multicolumn{2}{|c|}{ Sim } \\
\hline & & $\mathbf{n}$ & $\%$ \\
\hline \multirow{8}{*}{ Sentimentos positivos } & Compaixão & 36 & 52,2 \\
\hline & Aceitação & 18 & 26,1 \\
\hline & Tranquilidade & 13 & 18,8 \\
\hline & Satisfação & 7 & 10,1 \\
\hline & Alegria & 4 & 5,8 \\
\hline & Conforto & 3 & 4,3 \\
\hline & Bem-estar & 2 & 2,9 \\
\hline & Contentamento ou Felicidade & 2 & 2,8 \\
\hline \multirow{15}{*}{ Sentimentos negativos } & Insegurança & 29 & 42,0 \\
\hline & Tristeza & 28 & 40,6 \\
\hline & Estresse & 26 & 37,7 \\
\hline & Medo & 23 & 33,3 \\
\hline & Desconforto & 22 & 31,9 \\
\hline & Receio & 19 & 27,5 \\
\hline & Curiosidade & 19 & 27,5 \\
\hline & Ansiedade & 18 & 26,1 \\
\hline & Mal-estar / Insatisfação & 13 & 18,7 \\
\hline & Nervosismo & 11 & 15,9 \\
\hline & Raiva / Tédio & 7 & 10,1 \\
\hline & Confusão & 5 & 7,2 \\
\hline & Indiferença & 2 & 2,9 \\
\hline & Recusa & 2 & 2,9 \\
\hline & Vergonha & 1 & 1,4 \\
\hline
\end{tabular}

\section{DISCUSSÃO}

O presente estudo buscou avaliar atitudes, conhecimentos, percepções e sentimentos de técnicos de enfermagem que atuam em uma unidade de urgência e emergência sobre cuidados a pessoas com TM. Apesar da literatura ser escassa, o que pode ser observado tanto empiricamente quanto em estudos científicos, a assistência de enfermagem a pacientes com TM ainda encontra barreiras, profissionais de enfermagem apresentam percepções e sentimentos estigmatizantes, além de conhecimentos teóricopráticos insuficientes, o que pode resultar em atitudes negativas durante a assistência oferecida às pessoas diagnosticadas com TM.

As características sociodemográficas predominantes na amostra investigada foram: profissionais de enfermagem do sexo feminino, adultos, casados, com Ensino Médio e Superior, que não possuíam outro emprego. Há de se destacar que o predomínio de mulheres reflete uma particularidade importante identificada no exercício da enfermagem, que reflete a própria história da profissão ${ }^{(13-14)}$. Estudos apontam 
que a força de trabalho adulta ainda é predominantemente feminina, com forte associação entre o trabalho feminino e cuidados de saúde ${ }^{(13-17)}$. Estudo conduzido sobre as relações de gênero na enfermagem corrobora a produção científica do cuidado, evidenciada em trabalhos que privilegiam as experiências e vivências de trabalhadores do sexo feminino(15).

Por ser uma amostra de profissionais adultos e com nível de escolaridade entre Ensino Médio e Superior, é possível ampliar as possibilidades de aprendizado e educação continuada, que podem contribuir para aperfeiçoar experiência prática na temática em questão. Ainda que a maioria possua o nível médio, que é a escolaridade mínima exigida para a realização do curso técnico no Brasil, é importante assinalar que quase a metade $(46,4 \%)$ dos profissionais completou a graduação em Enfermagem, o que sugere que esses trabalhadores buscaram melhorar sua formação e competência técnica na profissão, podendo ainda estar à procura de novas oportunidades.

Quanto ao tempo de trabalho na instituição, os profissionais apresentaram ampla experiência profissional: $44,9 \%$ se dedicaram ao exercício da enfermagem entre dois a cinco anos, 39,1\% no PSM e 43,5\% na mesma equipe. Com base nesses dados pode-se esperar uma boa experiência e domínio na área do conhecimento. Pode-se deduzir ainda que esses profissionais sejam capazes de oferecer uma assistência profissionalmente qualificada aos pacientes com TM. Além disso, estudo mostrou que a escolaridade e o tempo em um mesmo serviço permitem a aquisição de novos conhecimentos na área de atuação profissional $^{(3)}$.

Apenas um estudo descreveu os temas que são ensinados na formação dos técnicos de enfermagem sobre dados epidemiológicos e clínicos relacionados aos TM. Esses temas ainda estão muito aquém do desejado, outras vezes são ausentes na maioria dos cursos técnicos de enfermagem, considerando que nos currículos essa disciplina possui uma carga horária reduzida ${ }^{(18)}$.

$\mathrm{Na}$ assistência de enfermagem às pessoas com TM, a relação terapêutica com foco na empatia tem sido considerada como a essência do trabalho do profissional de enfermagem. Esse tema pode não estar sendo contemplado nos conteúdos ministrados nos cursos de formação profissional, nem sequer considerado como necessário para a garantia de uma assistência mínima aos pacientes com sofrimento mental. Isso é efetivamente preocupante e exige das escolas formadoras uma atenção especial. Vale ressaltar que não há o propósito de direcionar o conhecimento em psiquiatria para a especialidade, mas enfocar o problema como uma responsabilidade de todo e qualquer profissional de saúde mental, no caso, os profissionais de enfermagem, respeitando os níveis de complexidade das situações.

Tem-se a convicção de que pessoas com TM não devam ser atendidas apenas pelos profissionais mais capacitados. Os demais profissionais podem e devem participar da assistência, pois, segundo a literatura, esses pacientes podem estar presentes em todos os setores de um serviço de saúde ${ }^{(12)}$. Desse modo, o afastamento dos profissionais com menor qualificação não se justifica, ao menos no nível do conhecimento dos participantes deste estudo, uma vez que a maioria recebeu conteúdos a respeito dos transtornos psiquiátricos e dos problemas relacionados. Portanto, teoricamente, possuíam algum nível de capacitação 
para a assistência.

Quanto aos conteúdos referentes à assistência de enfermagem aos pacientes com TM, deve ser considerada a necessidade de maiores investimentos na educação continuada e no oferecimento regular de recursos teóricos e supervisão, que vão além da identificação de problemas, incluindo as possíveis abordagens de acolhimento e a sistematização da assistência de enfermagem ${ }^{(3)}$.

Os resultados mostram que há uma substancial concordância entre os profissionais de que os cuidados de enfermagem em saúde mental deveriam estar integrados nos cuidados de saúde geral e que sem dúvida há necessidade de treinamentos e reciclagens sobre TM (Tabela 3). De acordo com o relatório da OMS, a integração da saúde mental nos cuidados de saúde de um modo geral é avaliada positivamente por oferecer amplos benefícios, incluindo a redução de custos nos cuidados de saúde e provimento de melhores cuidados aos pacientes psiquiátricos ${ }^{(5)}$. A OMS também reconhece que os recursos humanos fazem parte da força de trabalho nos serviços de saúde e incentiva a melhoria das competências técnicas na promoção da saúde mental e na prevenção, tratamento e manejo dos problemas relacionados aos TM valorizando essa medida, como uma estratégia possível de ser integrada em outros settings de atenção à saúde.

Outro dado interessante é que os profissionais de enfermagem concordaram que pessoas com TM são normalmente imprevisíveis, que necessitam de cuidados constantes, mas que podem levar uma vida normal. No entanto, $59,4 \%$ concordaram que as pessoas com sofrimento mental são normalmente perigosas, $37,5 \%$ consideram que elas podem ser violentas e $65,6 \%$ discordaram da noção de que essas pessoas poderiam ser capazes de receber tratamento no mesmo serviço de saúde que atende indivíduos com doença física (Tabela $3)$.

Esses achados se assemelham aos resultados de estudo que avaliou as percepções de profissionais da saúde em relação a pacientes internados com TM nas unidades do serviço ${ }^{(19)}$. Assim, as percepções são voltadas, principalmente, ao período em que os pacientes estão em crise ou estado crítico, além de serem percebidos como pessoas "diferentes", às vezes "violentas", "sem controle", "impulsivas", que "perderam a razão" e que podem ter limitações em seu processo adaptativo. Esses profissionais também percebem os pacientes como pessoas que precisam de ajuda por estarem vivenciando uma experiência de adoecimento psíquico e com graves dificuldades de se relacionarem devido aos seus comportamentos instáveis e inesperados. Além disso, são percebidos como pessoas que podem despertar nas "outras pessoas" sentimentos ambivalentes de "medo", "preconceito" e "compaixão"(19).

Evidências mostram que o comportamento agressivo é um dos sintomas comumente esperados em momentos de aguçamento dos sintomas (crise) nos diversos transtornos psiquiátricos e em algumas doenças neurológicas e clínicas, ou ainda, em pacientes sob efeito de substâncias psicoativas ${ }^{(20-22)}$. Estudo qualitativo avaliou as percepções de 27 membros de uma equipe de enfermagem de um pronto atendimento. Os resultados mostraram que os profissionais percebiam o paciente com TM como agressivo e agitado, demonstraram ter uma visão estereotipada e tinham muitas dúvidas sobre o cuidado de enfermagem mais apropriado frente a esses indivíduos, mas mesmo assim consideravam que eles necessitam de cuidados de 
enfermagem ${ }^{(23)}$.

Por outro lado, comportamentos violentos e agressivos exteriorizados por alguns pacientes provocam medo, ansiedade e insegurança generalizada, inclusive nos profissionais da equipe de enfermagem, de forma que os temores que alguns sentem em relação a "todos os pacientes psiquiátricos" é desproporcional aos poucos que, de fato, constituem risco para os outros. O medo excessivo nos profissionais pode prejudicar seu julgamento clínico e levar ao uso prematuro e em grandes quantidades de medicamentos sedativos e de restrição física, como as contenções no leito ${ }^{(24)}$.

Percepções e atitudes sobre a pessoa com sofrimento mental foram avaliadas também em outros grupos de profissionais de saúde (médicos e estudantes de medicina) de um hospital universitário em Londres. Os resultados mostraram que mais da metade dos participantes percebiam que os pacientes com dependência de álcool e/ou de outras drogas em comorbidade com esquizofrenia eram perigosos e imprevisíveis ${ }^{(25)}$.

Estudo menciona que, no decorrer da história, as pessoas com TM que não se enquadrassem nos padrões de normalidade vigentes em cada contexto histórico eram vítimas de preconceitos e estigmas por parte dos indivíduos considerados normais ${ }^{(9)}$. O preconceito é um juízo de valor baseado em premissa falsa, frequentemente sustentadas em mitos e estereótipos que conduzem à discriminação e estigmatizarão das pessoas identificadas como desviantes da norma. Esses são alguns dos fatores que embasaram a construção do conceito de doença mental e da pessoa com transtorno psíquico na história da psiquiatria, juntamente com as questões culturais ${ }^{(2,23)}$.

Na Tabela 3 nota-se ainda que a metade dos profissionais de enfermagem declarou se sentir confortável em atender pessoas com TM e 40,6\% consideraram o hospital como o local mais adequado para pessoas com TM morar. No entanto, metade dos profissionais discordou da assertiva de que se sentia à vontade em atender pacientes com TM e uma minoria $(17,2 \%)$ sentia-se indiferente em relação a esse assunto. Esses dados sugerem o desconhecimento sobre as opções na assistência dentro do sistema de saúde e o uso do modelo substitutivo de serviços de saúde mental que propõe o tratamento e a reabilitação fora dos muros hospitalares ${ }^{(17)}$. Como descrito na literatura ${ }^{(1,17)}$, a ideia da reabilitação psicossocial coincide com a necessidade de criar estratégias de ação que privilegiem as oportunidades de troca de recursos e afetos, com abertura de espaços de negociação para o paciente, sua família e sua rede social que estejam mais próximos da realidade e das implicações concretas do TM no cotidiano. O movimento da Reforma Psiquiátrica vem conquistando mudanças profundas na assistência em saúde mental preconizando a superação do modelo biomédico e hospitalocêntrico por uma rede de serviços comunitários, embora esta pouco tenha alcançado seu objetivo propriamente dito ${ }^{(17)}$.

No cotidiano da assistência à pessoa com TM, os profissionais de saúde, geralmente colocam todos os seus sentidos em funcionamento, o que faz aflorar seus sentimentos ${ }^{(18)}$. No presente estudo, os profissionais de enfermagem apresentaram sentimentos positivos de compaixão e aceitação em relação à pessoa com sofrimento mental, mas ainda foram muitos os sentimentos negativos (insegurança e tristeza) que parecem 
estar ainda enraizados nesses participantes (Tabela 4).

Há evidências de que os sentimentos e afetos que os membros da equipe de enfermagem das emergências psiquiátricas mais experimentam, ao trabalharem com o doente mental são relacionados ao medo, muitas vezes associado à possibilidade de sofrerem agressões físicas ${ }^{(24)}$, mas o sentimento de compaixão também aparece, geralmente ligado à disponibilidade interna para ajudar o paciente psiquiátrico, o que sugere maior compreensão dos diversos comportamentos paradoxais apresentados ${ }^{(18)}$.

Vale mencionar que a escassez de conhecimentos específicos em saúde mental contribui fortemente para a manifestação de sentimentos negativos como frustração, medo e angústia por parte dos profissionais de enfermagem que atuam em serviços de emergência e que comprometem a qualidade da assistência. Nesse contexto, estudo mostra a importância de incluir ações como educação permanente e aprimoramento na qualificação dos profissionais de enfermagem que atuam no atendimento às emergências psiquiátricas, a fim de proporcionar melhor cuidado e tratamento aos usuários desses serviços. Porém, para a efetivação dessas propostas ainda existem diversas lacunas a serem preenchidas, tais como a necessidade de mobilização dos gestores da saúde, motivação dos profissionais, elaboração de instrumentos de avaliação das estratégias a serem implementadas e a consolidação de uma rede de atenção em saúde mental integrada e humanizada ${ }^{(25)}$.

Estudo mostra que os pacientes percebidos como doentes mentais apresentam frequentemente problemas de comportamentos (como agressividade), solicitam muito a equipe de enfermagem e apresentam-se aos serviços agitados, delirantes ou com alucinações ${ }^{(2)}$. Essas percepções, baseadas em certa medida em situações vivenciadas de forma reinterada nos serviços, podem desencadear sentimentos ambíguos, desagradáveis e conflitantes nos profissionais de enfermagem, levando-os a adotarem julgamentos de valor que incentivam o aparecimento de posturas rígidas e punitivas em relação ao paciente. Entretanto, o lado racional lembra que se trata de um doente e que, portanto, deve ser ajudado, porém não sabendo lidar com certas situações, o profissional que não se posiciona de modo tecnicamente capaz frente aos problemas pode se omitir, e evitar o contato genuíno com os pacientes.

\section{CONCLUSÃO}

A possibilidade de avaliar conhecimentos, atitudes e percepções sobre saúde mental de profissionais de enfermagem tem sido considerada de crucial importância para o direcionamento de estratégias educacionais a serem implementadas de forma continuada, que possam levá-los a repensar valores, estigmas e preconceitos, conscientizando-os do necessário respeito para com o ser humano que apresenta sofrimento psíquico grave e persistente. Isso é valido para qualquer tipo de clientela ou especialidade, pois essa virtude depende da consciência de cada trabalhador de saúde nas suas ações sociais e atitudes pessoais como cidadão que se move dentro de um sistema em constante transformação.

Grande parte dos profissionais de enfermagem que participaram do presente estudo, por mais que tivessem recebido conteúdos sobre TM, ainda não se sentiam capacitados para trabalhar com esses 
pacientes nos serviços de urgência e emergência, em decorrência da pouca capacitação voltada a essa temática, o que coloca em relevo a existência de barreiras que impedem o desenvolvimento de ações de cuidado voltadas a essa população.

Uma limitação do presente estudo refere-se à falta de instrumentos padronizados e validados para avaliar conhecimentos e atitudes desses profissionais, principalmente relacionados às urgências e emergências, ou mesmo conteúdos específicos para enfermagem psiquiátrica disponibilizados nos cursos técnicos de enfermagem. Isso aponta para a importância de se desenvolverem ferramentas que sejam capazes de avaliar conhecimentos teórico-práticos de profissionais de nível técnico na área de saúde mental.

\section{REFERÊNCIAS}

1. Saraceno B, van Ommeren M, Batniji R, Cohen A, Gureje O, Mahoney J, et al. Barriers to improvement of mental health services in low-income and middle-income countries. Lancet [Internet]. 2007 [acesso em: 31 mar. 2016];370(9593):1164-74. Disponível em: http://dx.doi.org/10.1016/S0140-6736(07)61263-X.

2. Bennett E. Effects of a mental health-training program on health care workers' knowledge, attitude, and practice in Belize [dissertação]. Lisboa: Faculdade de Ciências Medicas/UNL; 2012

3. Junqueira MAB, Santos MA, Pillon SC. Competências atitudinais de estudantes de enfermagem na assistência a usuários de álcool. Rev Enferm Atenção Saúde [Internet]. 2014 [acesso em: 31 mar. 2016];3(2):76-85. Disponível em: http://www.uftm.edu.br/revistaeletronica/index.php/enfer/article/view/1022.

4. Happell B, Gaskin CJ. The attitudes of undergraduate nursing students towards mental health nursing: a systematic review. J Clin Nurs [Internet]. 2013 [acesso em: 31 mar. 2016];22(1-2):148-58. Disponível em:

http://dx.doi.org/10.1111/jocn.12022.

5. Organização Mundial de Saúde. Integração da saúde mental nos cuidados de saúde primários: uma perspectiva global [Internet]. Lisboa: OMS/WONCA; 2008 [acesso em: 31 mar. 2016]. Disponível em:

http://www.who.int/eportuguese/publications/Integracao_saude_mental_cuidados_primarios.pdf.

6. Tsai YF, Tsai MC, Lin YP, Weng CE, Chen CY, Chen MC. Facilitators and barriers to intervening for problem alcohol use. J Adv Nurs [Internet]. 2010 [acesso em: 31 mar. 2016];66(7):1459-68. Disponível em:

http://dx.doi.org/10.1111/j.1365-2648.2010.05299.x.

7. Prince A, Nelson K. Educational needs of practice nurses in mental health. J Prim Health Care [Internet]. 2011 [acesso em: 31 mar. 2016];3(2):142-9. Disponível em: http://www.publish.csiro.au/nid/315/paper/HC11142.htm. 8. Evans-Lacko S, Little K, Meltzer H, Rose D, Rhydderch D, Henderson C, et al. Development and psychometric properties of the Mental Health Knowledge Schedule. Can J Psychiatry [Internet]. 2010 [acesso em: 31 mar. 2016];55(7):440-8. Disponível em: http://publications.cpa-apc.org/media.php?mid=996.

9. Waidman MAP, Costa B, Paiano M. Community health agents' perceptions and practice in mental health. Rev Esc Enferm USP [Internet]. 2012 [acesso em: 31 mar. 2016];46(5):1170-7. Disponível em:

http://dx.doi.org/10.1590/S0080-62342012000500019.

10. Mion JZ, Schneider JF. Leitos Psiquiátricos em hospital geral: visão de profissionais que atuam em hospital geral. Rev. Eletr. Enf. [Internet]. 2003 [acesso em: 31 mar. 2016];5(1):38-42. Disponível em:

http://dx.doi.org/10.5216/ree.v5i1.771.

11. Cánovas Rodríguez JM, Hernández Ortega RC. Intervención de enfermería ante la agitación de una persona discapacitada intelectual institucionalizada. Enferm Glob. [Internet]. 2008 [acesso em: 31 mar. 2016];7(3). Disponível em: http://revistas.um.es/eglobal/article/view/36051.

12. Barros MA, Pillon SC. Atitudes dos profissionais do Programa Saúde da Família frente ao uso e abuso de drogas. Esc Anna Nery [Internet] 2007 [acesso em: 31 mar. 2016];11(4):655-62. Disponível em: http://dx.doi.org/10.1590/S1414-81452007000400016.

13. Minzoni MA. Assistência ao doente mental internado. Análise de uma experiência de treinamento de atendentes em hospital psiquiátrico. Bol Of Sant Panam [Internet]. 1980 [acesso em: 31 mar. 2016];88(3):242-51. Disponível em: http://iris.paho.org/xmlui/handle/123456789/17247. 
14. Filizola CLA, Milioni DB, Pavarini SCI. A vivência dos trabalhadores de um CAPS diante da nova organização do trabalho em equipe. Rev. Eletr. Enf. [Internet]. 2008 [acesso em: 31 mar. 2016];10(2):491-503. Disponível em: http://dx.doi.org/10.5216/ree.v10i2.8061.

15. Göttems LBD, Alves ED, Sena RR. Brazilian nursing and professionalization at technical level: a retrospective analysis. Rev Lat Am Enfermagem [Internet] 2007 [acesso em: 31 mar. 2016];15(5):1033-40. Disponível em: http://dx.doi.org/10.1590/S0104-11692007000500023.

16. Ferreira-Furegato AR, Frari-Galera SA, Pillon SC, Ferreira-Santos JL, Araujo-Pitia AC, Cardoso L. Characterizing mental healthcare service teams. Rev Salud Publica (Bogota). [Internet]. 2010 [acesso em: 31 mar. 2016];12(5):732-43. Disponível em: http://dx.doi.org/10.1590/S0124-00642010000500004.

17. Garla CC. Perfil dos profissionais de ambulatórios de saúde mental e suas práticas e opiniões sobre as políticas [dissertação]. Ribeirão Preto: Escola de Enfermagem de Ribeirão Preto/USP; 2010. 88 p.

18. Campos CJG. Teixeira MB. O atendimento do doente mental em pronto-socorro geral: sentimentos e ações dos membros da equipe de enfermagem. Rev Esc Enferm USP [Internet]. 2001 [acesso em: 31 mar. 2016];35(2):141-9. Disponível em: http://dx.doi.org/10.1590/S0080-62342001000200008.

19. Lauermann JD, Borges ZN. Saúde mental e hospitalização: qual a percepção dos profissionais da saúde? Rev Psicol da UNESP [Internet]. 2014 [acesso em: 31 mar. 2016];13(1):11-20. Disponível em:

http://pepsic.bvsalud.org/scielo.php?script=sci_arttext\&pid=S1984-90442014000100002\&lng=pt\&nrm=iso\&tlng=pt.

20. Mantovani C, Migon MN, Alheira FV, Del-Ben CM. Manejo de paciente agitado ou agressivo. Rev Bras Psiquiatr [Internet].; 2010 [acesso em: 31 mar. 2016];32 supl. 2:S96-103. Disponível em: http://dx.doi.org/10.1590/S151644462010000600006.

21. Paes MR, Maftum MA, Mantovani MF. Cuidado de enfermagem ao paciente com comorbidade clínico-psiquiátrica em um pronto atendimento hospitalar. Rev Gaucha Enferm [Internet]. 2010 [acesso em: 31 mar. 2016];31(2):277-84. Disponível em: http://dx.doi.org/10.1590/S1983-14472010000200011.

22. Kondo EH, Vilella JC, Borba LO, Paes MR, Maftum MA. Abordagem da equipe de enfermagem ao usuário na emergência em saúde mental em um pronto atendimento. Rev Esc Enferm USP [Internet]. 2011 [acesso em: 31 mar. 2016];45(2):501-7. Disponível em: http://dx.doi.org/10.1590/S0080-62342011000200028.

23. Mukherjee R. The stigmatisation of psychiatric illness: the attitudes of medical students and doctors in a London teaching hospital. Psychiatr Bull [Internet]. 2002 [acesso em: 31 mar. 2016];26(5):178-81. Disponível em:

http://dx.doi.org/10.1192/pb.26.5.178.

24. Henderson C, Thornicroft G. Stigma and discrimination in mental illness: time to Change. Lancet [Internet]. 2009 [acesso em: 31 mar. 2016];373(9679):1928-30. Disponível em: http://dx.doi.org/10.1016/S0140-6736(09)61046-1. 25. Ikuta CY, Santos MA, Badagnan HF, Donato ECSG, Zanetti ACG. Conhecimento dos profissionais de enfermagem em situações de emergência psiquiátrica: revisão integrativa. Rev. Eletr. Enf. [Internet]. 2013 [acesso em: 31 mar. 2016];15(4):1034-42. Disponível em: http://dx.doi.org/10.5216/ree.v15i4.20954. 